ISSN 1991-8631

Original Paper

http://indexmedicus.afro.who.int

\title{
Spéciation chimique des éléments traces métalliques dans un environnement lagunaire confiné : la baie de Bietry
}

\author{
Aoua S. COULIBALY *, Sylvain MONDE, Alexis Y. N'GUESSAN et Kouamé AKA \\ Université de Cocody, UFR-STRM, Département de Géosciences Marines, 22 B.P. 582 Abidjan 22, \\ Côte d'Ivoire. \\ *Auteur correspondant, E-mail : aouasougo@yahoo.fr
}

\section{RESUME}

L'analyse des concentrations métalliques déterminées pour les sédiments de la baie de Biétry a permis d'évaluer le niveau de pollution. Les métaux non pollués (classe 0 et 1) sont : Th, $\mathrm{Co}, \mathrm{V}, \mathrm{Ni}, \mathrm{Ag}$ et $\mathrm{Sb}$, avec des proportions qui varient de 55 à $70 \%$. Les métaux pollués (classes $2,3,5,6$ ) présentent des proportions variant de 100 à 70\%. Il s'agit de $\mathrm{Cr}, \mathrm{Mo}, \mathrm{As}, \mathrm{U}, \mathrm{Pb}, \mathrm{Cd}, \mathrm{Zn}$ et $\mathrm{Cu}$. Les résultats des extractions sélectives effectuées ont permis de déterminer le potentiel de mobilité et de disponibilité biologique de ces ETM (Eléments Traces Métalliques) polluants. Ainsi le gradient de biodisponibilité dans la baie de Biétry s'établit comme suit: $\mathrm{Ni}>\mathrm{Co}>\mathrm{Zn}$ et dans une moindre mesure $\mathrm{V}>\mathrm{Cu}>\mathrm{As}>\mathrm{Th}>\mathrm{Mo}>\mathrm{Pb}>\mathrm{Sb}$. Dans les phases porteuses des ETM, la fraction résiduelle est la plus dominante pour tous les métaux à l'exception de $\mathrm{Zn}$ et Mo. Du polluant le plus mobile au moins mobile, nous avons obtenu l'ordre suivant : $\mathrm{Zn}, \mathrm{Ni}, \mathrm{Pb}, \mathrm{Co}$, Th, $\mathrm{Mo}, \mathrm{As}, \mathrm{Sb}, \mathrm{Cr}$.

() 2011 International Formulae Group. All rights reserved.

Mots clés : Igeo, pollution, biodisponibilité, spéciation métallique.

\section{INTRODUCTION}

Les éléments traces sont des constituants de la croûte terrestre, dont la concentration est pour chacun d'entre eux inférieure à 100 ppm (Baize, 1997). Ils ne représentent que $0,6 \%$ du total des éléments chimiques de la croûte terrestre. Cependant, leurs effets sur les écosystèmes sont souvent dévastateurs. Parmi ces éléments, certains, appelés oligoéléments, s'avèrent indispensables à l'activité biologique à des concentrations raisonnables $(\mathrm{Cu}, \mathrm{Zn}, \mathrm{Ni}, \mathrm{Cr}$, $\mathrm{Co}, \mathrm{V}, \mathrm{Mo}, \mathrm{Sb}$, Th, U, Ag). A l'inverse d'autres éléments, tels que $\mathrm{Pb}, \mathrm{Cd}$, As, malgré leur affinité avec les groupes $-\mathrm{SH}$ et $-\mathrm{NH}$ dans les enzymes, ne présentent pas de fonction biologique reconnue jusqu'à présent et sont par conséquent considérés comme particulièrement dangereux et toxiques pour les écosystèmes, ainsi que pour l'homme (Sigg et al., 2006). Ces éléments parviennent dans l'environnement grâce à deux processus : l'un naturel, caractérisé par l'altération des roches, la diffusion des aérosols naturels à partir du sol et de l'eau; et l'autre anthropique englobant les apports industriels, dominés principalement par les rejets des industries métallurgiques, les apports agricoles (fertilisants, pesticides, etc.) et domestiques (Salvarredy-Aranguren et al., 2008). 
En Côte d'Ivoire, dans la région d'Abidjan, les rejets urbains et industriels se concentrent dans la baie de Biétry. C'est le point d'aboutissement principal du réseau d'égouts dans le secteur Sud d'Abidjan depuis 1984. Cet environnement confiné montre un phénomène d'eutrophisation et périodiquement, des morts massives de poissons. L'analyse des métaux des sédiments s'impose afin de mieux cerner les processus qui s'y déroulent. A cet effet, deux (2) approches géochimiques présentent un intérêt indéniable dans l'analyse des ETM (Eléments Traces Métalliques) des sédiments superficiels de la baie de Biétry :

- D'un point de vue environnemental, la spéciation traduit la distribution des espèces chimiques d'un élément donné dans un hydrosystème (Templeton et al., 2000). Tous ces métaux sont différemment réactifs, toxiques ou biodisponibles pour le biota (Gómez-Ariza et al., 2001). De ce fait, la mesure de la concentration totale des ions métalliques en solution n'apporte que peu d'informations pour la prédiction du comportement métallique (Filella et al., 1995). C'est alors qu'une analyse de spéciation, selon I'IUPAC (International Union for Pure and Applied Chemistry), correspondant à l'activité analytique d'identification et de mesure de la quantité d'une ou plusieurs espèces chimiques individuelles dans un échantillon (Templeton et al., 2000), s'impose. Toutefois, compte tenu des procédures et techniques analytiques généralement utilisées, cette identification détermine des contenus de composés «extractibles» (fractionnement) plutôt que des espèces chimiques bien définies. Ainsi, certains auteurs (Quevauviller, 2002) emploient le terme d'«éléments traces extractibles » en relation avec un réactif défini, préférentiellement à celui de « spéciation ».

- Une autre approche essentielle dans les évaluations écologiques des pollutions métalliques est l'estimation de la toxicité potentielle d'un élément en fonction de sa forme chimique. Cette toxicité est en relation étroite avec la mobilité des composés métalliques qui dépend elle-même de leur spéciation (Batley, 1989).

Ainsi, de l'analyse de ces deux approches géochimiques, il ressort qu'une attention particulière devrait être accordée à l'étude des différentes phases porteuses. En effet, les éléments traces contenus dans la matrice cristalline des minéraux sont moins biodisponibles (moins susceptibles d'être mobilisés dans la phase dissoute) que ceux présents sur les sites superficiels d'adsorption des particules ou associés aux carbonates et à la matière organique.

La présente étude fait une analyse de la biodisponibilité et de la spéciation chimique des éléments traces métalliques des sédiments superficiels de la baie de Biétry. Cette étude fait l'état des lieux des teneurs en métaux des sédiments, évalue le degré de pollution et détermine la proportion des métaux disponibles et des différentes phases porteuses des métaux.

\section{MATERIEL ET METHODES \\ Echantillonnage}

Une campagne d'échantillonnage a été organisée en janvier 2006 dans la lagune Ebrié par l'Université d'Abidjan-Cocody, en collaboration avec le CIAPOL (Centre Ivoirien Anti-Pollution). Le prélèvement des sédiments s'est fait dans la baie de Bietry (Figure 1) selon un mode de choix des sites d'échantillonnage représentatif de l'ensemble de la baie. Au total une douzaine d'échantillons de sédiments de surface ont été prélevés à l'aide d'une benne Van Veen, à des stations positionnées par un récepteur GPS. Pour éviter toute contamination métallique due à la benne, les échantillons ont été recueillis au cœur de la benne avec des spatules en plastiques. Ils ont été conditionnés dans des flacons de $50 \mathrm{ml}$. Afin d'éviter toute pollution, tout le flaconnage en contact avec les échantillons de sédiments destinés aux dosages des ETM est soigneusement décontaminé à l'aide d'une solution de $\mathrm{HNO}_{3}$ (qualité «normapur ») à 10\% (v/v) pendant 
une semaine puis rincé cinq (5) fois à l'eau désionisée et cinq (5) fois à l'eau Milli-Q et séché sous une hotte à flux laminaire.

\section{Environnement et précautions analytiques}

Le dosage des ETM, avec des concentrations de l'ordre de quelques ng. $\mathrm{L}^{-1}$, a nécessité une prise de précautions particulières pour préserver les échantillons de toute contamination extérieure. Les manipulations et les dosages en laboratoire (laboratoire TGM, Université Bordeaux I) se sont déroulés dans des salles en surpression et équipés d'un système de filtration d'air (filtre MLK 6/12, 0,3 $\mu \mathrm{m}$ de porosité) assurant un environnement propre et minimisant les risques de contamination. Toute manipulation nécessite le port de gants et de blouses en polypropylène.

Tout le flaconnage utilisé au cours des préparations des réactifs et des dosages des ETM (flacons divers, embouts de pipettes, bombes de digestion,...) est en polypropylène ou téflon, matériaux résistant aux acides et minimisant toutes réactions d'adsorption ou désorption de métaux au niveau des parois. Avant toute utilisation, ce flaconnage a été décontaminé selon le protocole précédemment décrit pour le flaconnage utilisé sur le terrain.

Les produits chimiques de qualité «ultrapure» et «suprapure» ont été préférentiellement utilisés. Les pesées de la fraction solide ont été effectuées à l'aide d'une balance Sartorius MC 210 S de précision $\pm 0,1 \mathrm{mg}$.

\section{Choix de la procédure d'extraction}

Les techniques de spéciation peuvent être regroupées en deux catégories : (i) celles où des fractions opérationnellement définies du métal total sont détectées, et (ii) celles mesurant les concentrations métalliques totales (Filella et al., 1995). Les techniques utilisées dans le cadre de notre étude appartiennent à la deuxième catégorie. En effet, les méthodes de détection que nous avons employées (i.e. ICP-MS, spectrométrie d'absorption atomique) ne mesurent que les concentrations totales et doivent donc être couplées avec des techniques préalables de séparation pour extraire les différents phases sous lesquelles l'élément à doser est présent dans l'échantillon.

La répartition des métaux (i.e. leur «forme» chimique) dans les sédiments détermine leurs comportements dans l'environnement. L'extraction chimique sélective est le moyen le plus largement répandu pour déterminer cette partition. La majorité des procédures d'extractions décrites dans la littérature porte sur la distribution des métaux dans les différentes phases du sédiment : métaux échangeables, carbonates, fraction réductible (hydroxydes de $\mathrm{Fe}$ et $\mathrm{Mn}$ ) et oxydable (matière organique et sulfures) et minéraux résiduels. Ces extractions sélectives peuvent être menées suivant deux procédés distincts : (i) extractions séquentielles (utilisation d'un seul et même échantillon que l'on soumet successivement à des réactifs de force croissante) et (ii) extractions parallèles (utilisation d'aliquotes d'un même échantillon pour chaque attaque).

Pour réaliser les extractions sélectives sur nos échantillons de sédiments, nous avons choisi de mettre en place une procédure d'extractions sélectives parallèles plutôt que séquentielles. La méthode «parallèle » permet de s'affranchir d'un certain nombre de problèmes et d'inconvénients inhérents aux extractions séquentielles (Farrah et Pickering, 1993). Ces problèmes sont : (i) possibles changements de la spéciation d'un élément au cours des étapes successives d'extraction et de rinçage de l'échantillon après chaque étape (Rosenberg et Ariese, 2001) ; (ii) risques multiples de contamination de l'échantillon par l'utilisation successives de réactifs (Quevauviller, 1998). Toutes ces raisons peuvent être responsables de l'altération de l'information originellement présente dans l'échantillon. De plus, les avantages de l'approche «parallèle » incluent : un faible risque de perte d'échantillon pendant l'extraction ; une erreur ou un problème survenant pendant une extraction ne 
compromet pas l'intégralité de la procédure (Tack et al., 1996).

Notre procédure est basée principalement sur le schéma séquentiel conventionnel de Tessier et al. (1979). Des études (Alborés et al., 2000) ont montré que des résultats similaires d'extraction d'ETM $(\mathrm{Cr}, \mathrm{Ni}, \mathrm{Pb}, \mathrm{Cu}$ et $\mathrm{Zn}$ ) étaient obtenus en appliquant les différentes étapes du schéma séquentiel d'extractions de Tessier et al. (1979) dans une procédure d'extractions parallèles.

\section{Protocole d'extraction}

$\mathrm{Au}$ laboratoire, une douzaine de sédiments ont été mis à l'étuve $\left(50{ }^{\circ} \mathrm{C}\right)$ jusqu'à séchage complet. Ils ont été ensuite broyés et homogénéisés dans un mortier en agate manuel. Pour chaque extraction, des blancs de réactifs sont réalisés ; ces blancs sont ensuite soustraits des concentrations de métaux. Toutes les attaques sélectives sont conduites dans des tubes à centrifugation en polypropylène de $50 \mathrm{~mL}$ préalablement décontaminés. L'agitation des échantillons a été effectuée à l'aide d'un agitateur mécanique rotatif.

Après chaque extraction sélective, les échantillons sont centrifugés pendant $20 \mathrm{~min}$ à 5000 tr.min- ${ }^{1}$; le surnageant est ensuite prélevé et filtré à travers un filtre-seringue en polycarbonate de $0,2 \mu \mathrm{m}$ de porosité, et stocké au froid $\left(4^{\circ} \mathrm{C}\right)$ jusqu'à son analyse.

Extraction à l'acétate (Fraction 1)

L'utilisation de l'acétate a pour but d'extraire les métaux liés aux « carbonates ». La prise d'échantillon est de $500 \mathrm{mg}$ pour 10 mL d'acétate de sodium ( $\mathrm{NaOAc}, 1 \mathrm{M})$, ajusté à pH 5 avec de l'acide acétique. Des ajustements de $\mathrm{pH}$ sont réalisés par ajout de $500 \mu \mathrm{L}$ de HOAc $(5 \mathrm{M})$ après $1 \mathrm{~h}, 1 \mathrm{~h} 30$ et $2 \mathrm{~h} 30$ d'agitation. Orsini et Bermond (1993) ont signalé la nécessité de ses ajustements de $\mathrm{pH}$ autour de 5. En effet, lors de la dissolution de $\mathrm{CaCO}_{3}$, le $\mathrm{pH}$ de la solution augmente, avec comme conséquence la décroissance de la cinétique de dissolution.

\section{Extraction à l'ascorbate (Fraction 2)}

L'extraction à l'ascorbate permet de déterminer la fraction de métaux liés aux oxydes de fer et de manganèse. La solution d'attaque ascorbate est préparée en mélangeant dans 1 L d'eau Milli-Q $50 \mathrm{~g}$ d'hydrogénocarbonate de sodium $\left(\left(\mathrm{HCO}_{3}\right) \mathrm{Na}\right.$, solution tampon maintenant le $\mathrm{pH}$ de la solution à 8) et $50 \mathrm{~g}$ de citrate dihydrate de sodium $\left(\mathrm{C}_{6} \mathrm{H}_{5} \mathrm{Na}_{3} \mathrm{O}_{7} \cdot 2 \mathrm{H}_{2} \mathrm{O}\right.$, complexant des métaux en solution). On bulle la solution avec de l'azote pour éliminer l'oxygène dissous. Nous ajoutons ensuite $20 \mathrm{~g}$ d'acide ascorbique $\left(\left(\mathrm{C}_{6} \mathrm{H}_{8} \mathrm{O}_{6}\right)\right.$, réducteur des oxydes). Pour 200 $\mathrm{mg}$ d'échantillon, $12,5 \mathrm{~mL}$ de solution ascorbate sont ajoutés. Les échantillons sont ensuite agités en continue pendant $24 \mathrm{~h}$.

Extraction à l'eau oxygénée - $\mathrm{H}_{2} \mathrm{O}_{2}$ : Fraction 3

La fraction 3 contient les métaux liés à la matière organique et aux sulfures. La prise d'échantillon est de $1 \mathrm{~g}$, auquel on ajoute 8 $\mathrm{mL}$ de $\mathrm{H}_{2} \mathrm{O}_{2}$ à $30 \%$ (ajusté à $\mathrm{pH} 5$ avec $\mathrm{NaOH})$. Le mélange est chauffé à $85^{\circ} \mathrm{C}( \pm 2$ ${ }^{\circ} \mathrm{C}$ ) à l'étuve pendant $2 \mathrm{~h}$ avec des agitations intermittentes pour empêcher toute effervescence qui causerait une perte de matériel. On rajoute ensuite $3 \mathrm{~mL}$ de $\mathrm{H}_{2} \mathrm{O}_{2}$ (pH 5) et on chauffe à nouveau à $85{ }^{\circ} \mathrm{C}$ pendant $3 \mathrm{~h}$ en agitant par intermittence. Après refroidissement, $5 \mathrm{~mL}$ d'une solution d'acétate d'ammonium ((NH4OAc) 1M, complexant des métaux dissous) est ajouté puis l'échantillon est dilué avec de l'eau Milli$\mathrm{Q}{ }^{\circledR}$ jusqu'à $20 \mathrm{~mL}$. Le tout est ensuite agité pendant $30 \mathrm{~min}$.

\section{Extraction avec $\mathrm{HCl}$ (Fraction 4)}

Elle concerne les métaux liés à la fraction « réductible ». Une solution de $\mathrm{HCl}$ $1 \mathrm{~N}$ est préparée par dilution de $\mathrm{HCl}$ «suprapur» à 30,9\% dans de l'eau Milli-Q. La prise d'échantillon est de $200 \mathrm{mg}$ auquel on ajoute $12,5 \mathrm{~mL}$ de la solution de $\mathrm{HCl}$. Les échantillons sont agités en continue pendant 24h.

\section{Fraction 5}

Il s`agit de l'extraction de l'ensemble des métaux. Une quantité de sédiments (30 $\mathrm{mg})$ est digérée à l'eau régale $(750 \mu \mathrm{L} \mathrm{HCl}$ 
«suprapur» à $30,9 \%+250 \mu \mathrm{L} \quad \mathrm{HNO}_{3}$ «suprapur» à 65,5\%) et $2 \mathrm{~mL}$ de $\mathrm{HF}$ «suprapur» à $65 \%$ dans des flacons en téflon fermés hermétiquement et maintenus pendant $2 \mathrm{~h}$ à $110^{\circ} \mathrm{C}$ sur une plaque chauffante. Après refroidissement, les gouttelettes condensées sur les bouchons des flacons ont été récupérées à l'aide d'un rinçage à l'eau MilliQ. L'évaporation du contenu des flacons est réalisée par chauffage à $100{ }^{\circ} \mathrm{C}$ pendant $12 \mathrm{~h}$. On laisse refroidir de nouveau pour effectuer la reprise du résidu de digestion avec $250 \mu \mathrm{L}$ de $\mathrm{HNO}_{3}$ à $65,5 \%$ et $5 \mathrm{~mL}$ d'eau Milli-Q. La nouvelle solution est chauffée une dernière fois à $80{ }^{\circ} \mathrm{C}$ pendant $10 \mathrm{~min}$. Après refroidissement et décantation, $3,5 \mathrm{~mL}$ de surnageant est prélevé et conservé dans un flacon en polypropylène auquel on ajoute 6,5 mL d'eau Milli-Q.

\section{Spectrométrie de masse à plasma couplé (ICP-MS)}

Les mesures des ETM (V, Cr, Co, Ni, $\mathrm{Cu}, \mathrm{Zn}, \mathrm{Mo}, \mathrm{Ag}, \mathrm{As}, \mathrm{Cd}, \mathrm{Pb}$, Th et $\mathrm{U}$ ) ont été réalisées à l'aide d'un spectromètre de masse couplé à un plasma d'ionisation (Perkin-Elmer type Elan 5000) permettant de détecter des éléments en traces à des concentrations minimales de $10^{-3} \mu \mathrm{g} \cdot \mathrm{L}^{-1}$. Toutes les analyses ont été réalisées en mode quantitatif.

Tous les étalons et échantillons sont dilués dans une matrice $\mathrm{HNO}_{3}$ (qualité «ultrapure») à $1 \%$. La sensibilité de l'appareil est ajustée à l'aide d'une solution de $10 \mu \mathrm{g} . \mathrm{L}^{-1}$ d'isotopes couvrant une large gamme de masse $\left({ }^{207,208} \mathrm{~Pb},{ }^{24} \mathrm{Mg}\right.$ et $\left.{ }^{103} \mathrm{Rh}\right)$; le bruit de fond est mesuré à la masse 220. Le taux d'oxydes et le taux d'ions doublement chargés sont également vérifiés pour ne pas excéder $3 \%$ lors de nos dosages (typiquement autour de $2 \%$ ). Une calibration interne et externe est réalisée $\left(\mathrm{Co}=500 \mu \mathrm{g} . \mathrm{L}^{-1}, \mathrm{Cd}, \mathrm{Sn}, \mathrm{Mo}, \mathrm{Th}, \mathrm{U}\right.$ $\left.=50 \mu \mathrm{g} \cdot \mathrm{L}^{-1}\right)$. Une gamme de six (6) étalons, obtenue par dilution de la solution mère (1000 fois et 25 fois pour les limites basse et haute, respectivement) a été utilisée pour les différentes séries d'analyses. Les droites de calibration ainsi obtenues permettent de déterminer la concentration des éléments métalliques de nos échantillons avec un coefficient de corrélation supérieur à 0,995 . De plus, un étalon est mesuré tous les cinq (5) échantillons afin de déterminer la dérive de la sensibilité de l'appareil. Pour toutes les séries d'analyses, les blancs analytiques (eau Milli$\mathrm{Q}+\mathrm{HNO}_{3}$ ) sont mesurés et leur valeur soustraite des valeurs de concentration; pour les analyses concernant les attaques de la fraction solide, les valeurs des blancs d'attaques sont également retranchées.

\section{Déterminisme du bruit de fond et du niveau de pollution}

Compte tenu de l'absence voire de la rareté des données de concentrations métalliques dans la baie, un échantillon $\mathrm{A}$, a été prélevé dans la lagune Ebrié en marge de cette étude. Le choix de cet échantillon, qui sert de bruit de fond de la lagune, a été fait en fonction de sa localisation géographique (éloigné de toutes influences anthropiques) et conforté par les résultats de la composition chimique.

Pour la détermination du degré de pollution, l'indice de geoaccumulation ou Igeo (Müller, 1981) a été utilisé. Cet indice (Tableau 1) se détermine à partir du niveau de base de la teneur en métaux dans le sédiment selon la formule suivante :

$$
\text { Igeo }=\log _{2}\left(\mathrm{C}_{\mathrm{n}} / 1,5 \mathrm{~B}_{\mathrm{n}}\right)
$$

Avec : $C_{n}$ (Concentration dans le sédiment pour l'élément $n$ ), $B_{n}$ (Bruit de fond géochimique pour l'élément $n$ ) et 1,5 (Constante prenant en compte les fluctuations naturelles de la teneur d'une substance donnée dans un environnement et les faibles influences anthropiques).

\section{RESULTATS}

Les concentrations métalliques totales obtenues pour les sédiments de la baie de Biétry sont très hétérogènes. Ces valeurs varient aussi bien d'un point de vue qualitatif, quantitatif que spatiale. Le Tableau 2 présente les teneurs totales ainsi que les valeurs maximales, minimales, la moyenne et l'écart 
type. A l'analyse de ce tableau, nous notons que :

(i) les teneurs moyennes des oligoéléments qui s'avèrent indispensables à l'activité biologique varient entre 8 à 234 mg/l. Ce sont: $\mathrm{Cu}(25,72 \mathrm{mg} / \mathrm{l}), \mathrm{Zn}(137,33$ $\mathrm{mg} / \mathrm{l}), \mathrm{Ni}(20,04 \mathrm{mg} / \mathrm{l}), \mathrm{Cr}(223,06 \mathrm{mg} / \mathrm{l})$, $\operatorname{Co}(7,53 \mathrm{mg} / \mathrm{l}), \quad \mathrm{V}(48,18 \mathrm{mg} / \mathrm{l}), \quad$ Mo $(8,28$ $\mathrm{mg} / \mathrm{l}), \operatorname{Th}(3,31 \mathrm{mg} / \mathrm{l}), \mathrm{U}(3,32 \mathrm{mg} / \mathrm{l})$ et $\operatorname{Ag}(0,48$ $\mathrm{mg} / \mathrm{l})$.

(ii) les métaux reconnus comme étant dangereux ou toxiques pour les écosystèmes présentent quant à eux des moyennes comprises entre 0,50 et $49 \mathrm{mg} / \mathrm{l}$. Ce sont: $\mathrm{Pb}(48,86 \mathrm{mg} / \mathrm{l}), \mathrm{Cd}(0,50 \mathrm{mg} / \mathrm{l})$ et $\mathrm{As}(5,70$ $\mathrm{mg} / \mathrm{l})$.

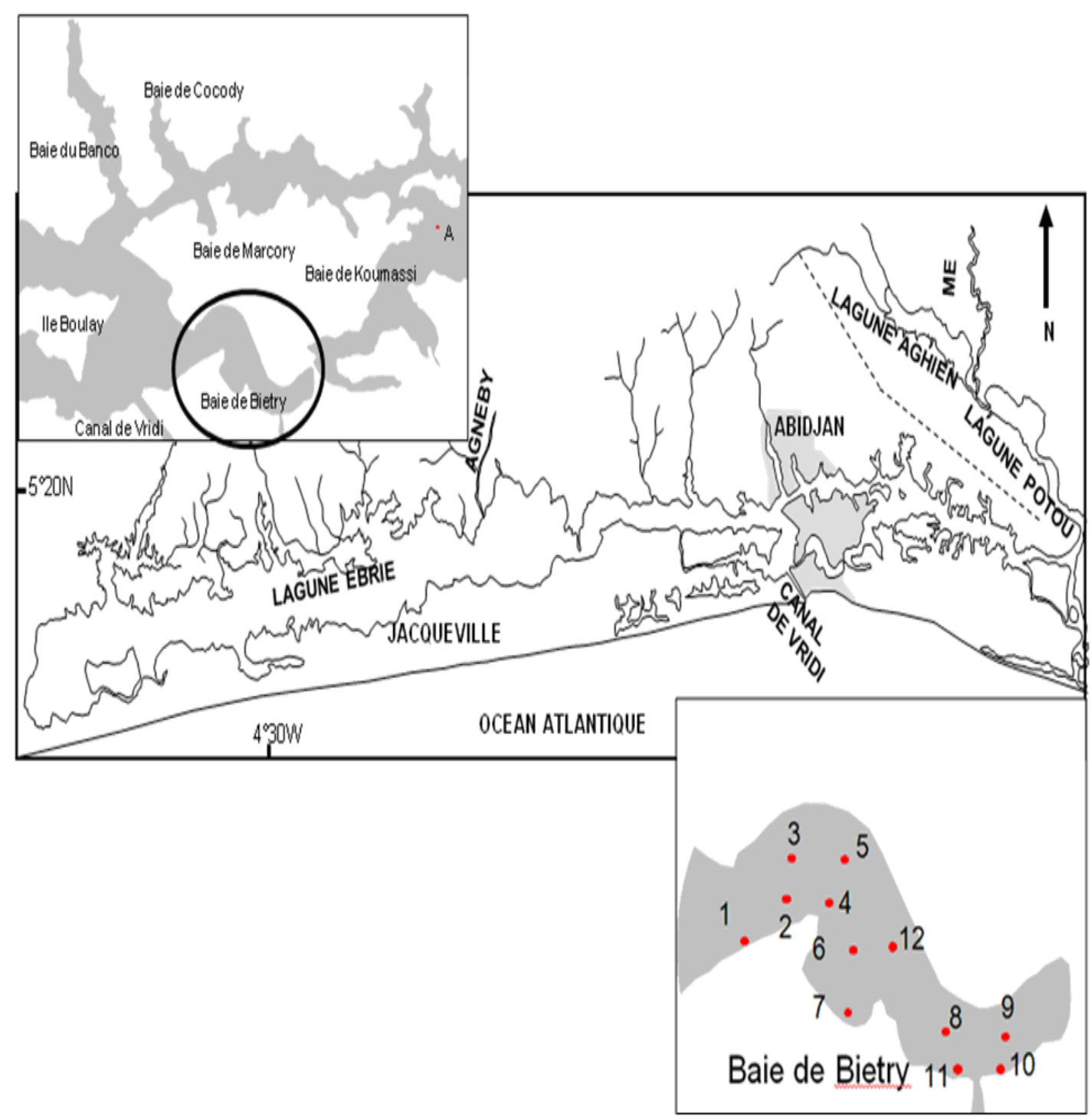

Figure 1 : Stations de prélèvement des sédiments dans la baie de Biétry (Lagune Ebrié). 


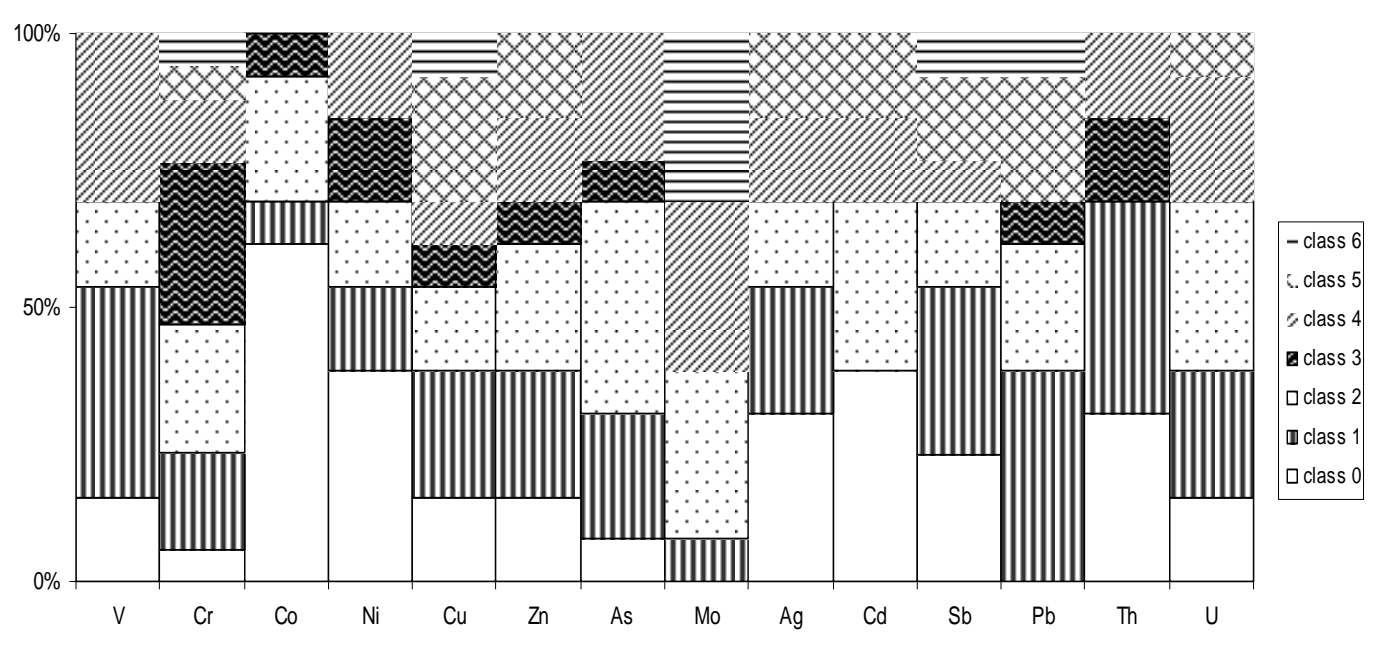

Figure 2: Proportion du niveau de pollution dans les sédiments de la baie de Biétry.
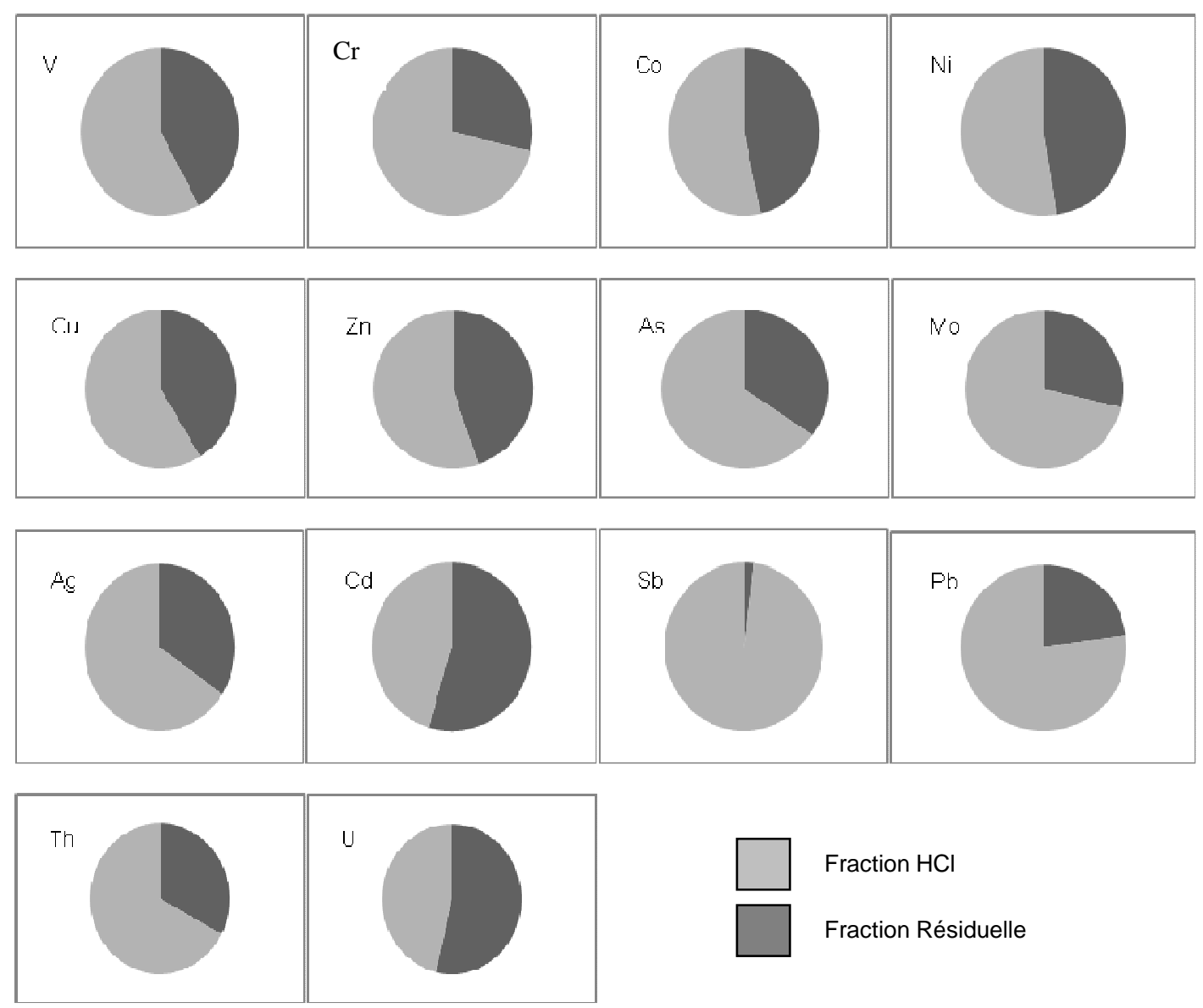

Fraction $\mathrm{HCl}$

Fraction Résiduelle

Figure 3 : Contribution moyenne des métaux de la fraction $\mathrm{HCl}$ à la teneur totale. 


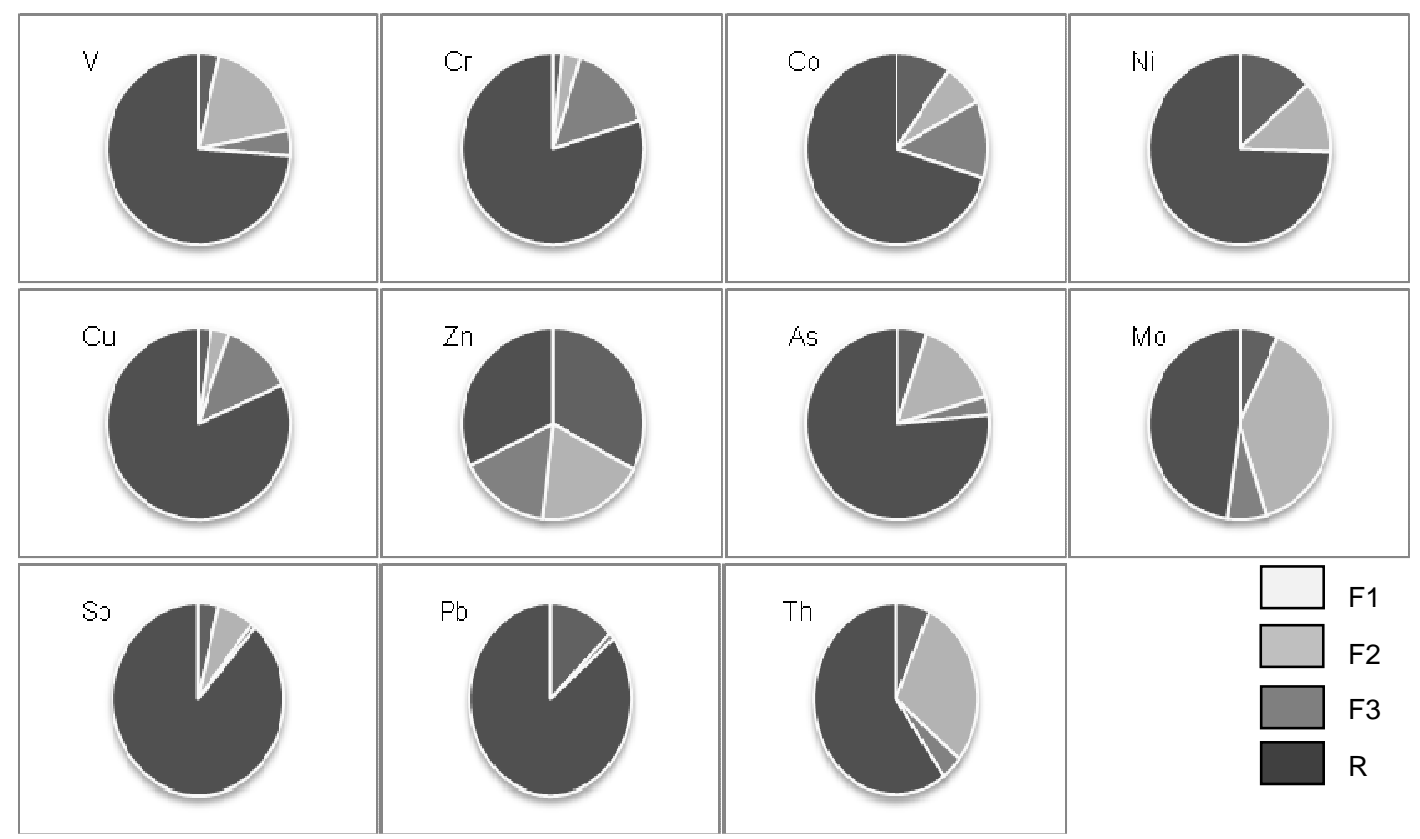

Figure 4: Proportions moyennes (\%) des fractions opérationnelles (F1, F2, F3 et R).

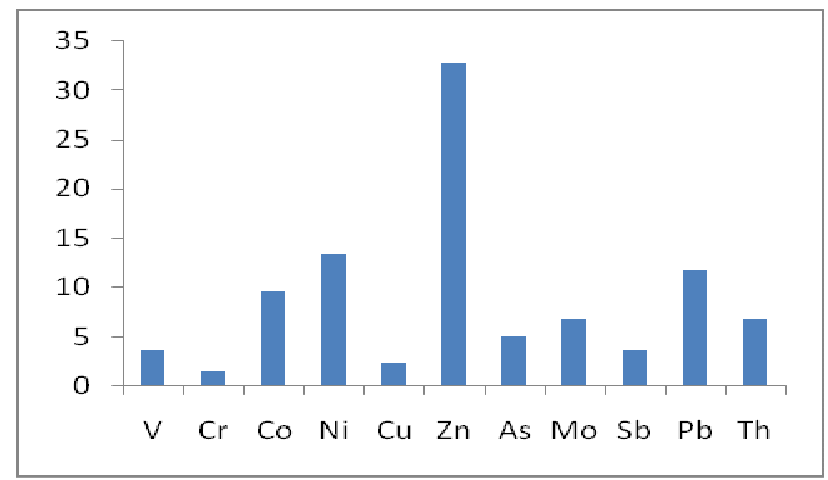

Figure 5 : Proportion (\%) des variations de la fraction acétate (F1).

Tableau 1 : Classification de Müller (1981).

\begin{tabular}{lll}
\hline Classe & Valeur & Intensité de pollution \\
\hline 0 & Igeo $\leq 0$ & Non pollué \\
1 & $0<$ Igeo $<1$ & De non pollué à modérément pollué \\
2 & $1<$ Igeo $<2$ & Modérément pollué \\
3 & $2<$ Igeo $<3$ & De modérément pollué à sévèrement pollué \\
4 & $3<$ Igeo $<4$ & Sévèrement pollué \\
5 & $4<$ Igeo $<5$ & De sévèrement pollué à très sévèrement pollué \\
6 & $5<$ Igeo & Très sévèrement pollué \\
\hline
\end{tabular}


A. S. COULIBALY et al. / Int. J. Biol. Chem. Sci. 5(6): 2543-2556, 2011

Tableau 2 : Concentrations totales $(\mathrm{mg} / \mathrm{kg})$ des sédiments.

\begin{tabular}{|c|c|c|c|c|c|c|c|c|c|c|c|c|c|c|}
\hline Echantillons & $\mathbf{V}$ & $\mathrm{Cr}$ & Co & $\mathrm{Ni}$ & $\mathbf{C u}$ & $\mathbf{Z n}$ & As & Mo & $\mathbf{A g}$ & Cd & $\mathrm{Sb}$ & $\mathbf{P b}$ & Th & $\mathbf{U}$ \\
\hline 1 & 23,62 & 54,79 & 3,78 & 20,58 & 18,25 & 87,27 & 2,92 & 1,71 & 0,14 & 0,16 & 0,17 & 19,42 & 1,99 & 1,43 \\
\hline 2 & 12,73 & 28,34 & 1,24 & 5,30 & 1,83 & 16,39 & 2,17 & 0,61 & 0,06 & 0,06 & 0,10 & 6,53 & 0,72 & 0,54 \\
\hline 3 & 103,87 & 130,87 & 21,50 & 52,18 & 43,56 & 264,32 & 16,79 & 13,61 & 0,76 & 1,12 & 1,01 & 73,14 & 8,47 & 6,12 \\
\hline 4 & 123,82 & 180,80 & 16,27 & 59,30 & 64,46 & 248,55 & 11,26 & 10,51 & 0,98 & 0,76 & 1,59 & 113,88 & 9,34 & 6,34 \\
\hline 5 & 8,05 & 9,22 & 1,57 & 3,50 & 2,70 & 18,85 & 0,91 & 0,51 & 0,08 & 0,07 & 0,11 & 4,72 & 0,73 & 0,83 \\
\hline 6 & 18,19 & 14,58 & 2,78 & 3,57 & 2,80 & 27,24 & 1,47 & 0,41 & 0,07 & 0,07 & 0,09 & 4,91 & 0,80 & 0,86 \\
\hline 7 & 25,82 & 25,84 & 5,53 & 13,30 & 6,67 & 53,01 & 3,05 & 1,89 & 0,14 & 0,22 & 0,19 & 11,45 & 2,00 & 2,10 \\
\hline 8 & 7,95 & 11,08 & 3,43 & 5,01 & 2,75 & 25,23 & 1,15 & 0,63 & 0,07 & 0,07 & 0,07 & 5,39 & 0,62 & 0,48 \\
\hline 9 & 17,59 & 36,31 & 3,90 & 6,00 & 6,13 & 51,29 & 3,19 & 2,05 & 0,20 & 0,21 & 0,16 & 10,87 & 1,26 & 1,22 \\
\hline 10 & 99,91 & 2058,6 & 10,31 & 31,04 & 79,09 & 498,51 & 5,92 & 35,09 & 1,56 & 1,39 & 1,54 & 209,04 & 5,92 & 11,53 \\
\hline 11 & 16,98 & 24,95 & 3,94 & 4,89 & 9,96 & 48,94 & 2,41 & 2,85 & 0,21 & 0,24 & 0,19 & 12,14 & 1,20 & 1,75 \\
\hline 12 & 119,67 & 101,21 & 16,09 & 35,77 & 70,40 & 308,32 & 17,18 & 29,51 & 1,50 & 1,69 & 13,73 & 114,79 & 6,64 & 6,64 \\
\hline Min & 7,95 & 9,22 & 1,24 & 3,50 & 1,83 & 16,39 & 0,91 & 0,41 & 0,06 & 0,06 & 0,07 & 4,72 & 0,62 & 0,48 \\
\hline Max & 123,82 & 2058,6 & 21,50 & 59,30 & 79,09 & 498,51 & 17,18 & 35,09 & 1,56 & 1,69 & 13,73 & 209,04 & 9,34 & 11,53 \\
\hline Moyenne & 48,18 & 223,06 & 7,53 & 20,04 & 25,72 & 137,33 & 5,70 & 8,28 & 0,48 & 0,50 & 1,58 & 48,86 & 3,31 & 3,32 \\
\hline Ecart type & 47,68 & 580,60 & 6,82 & 20,01 & 29,95 & 155,61 & 5,97 & 12,04 & 0,57 & 0,58 & 3,87 & 65,67 & 3,30 & 3,51 \\
\hline
\end{tabular}


A. S. COULIBALY et al. / Int. J. Biol. Chem. Sci. 5(6): 2543-2556, 2011

Tableau 3 : Concentrations moyennes $(\mathrm{mg} / \mathrm{kg})$ des sédiments et UCC.

\begin{tabular}{|c|c|c|c|c|c|c|c|c|c|c|c|c|c|c|}
\hline Echantillons & $\mathbf{V}$ & $\mathrm{Cr}$ & Co & $\mathbf{N i}$ & $\mathbf{C u}$ & $\mathbf{Z n}$ & As & Mo & Ag & Cd & $\mathbf{S b}$ & $\mathbf{P b}$ & Th & $\mathbf{U}$ \\
\hline Moyenne & 48,18 & 223,06 & 7,53 & 20,04 & 25,72 & 137,33 & 5,70 & 8,28 & 0,48 & 0,50 & 1,58 & 48,86 & 3,31 & 3,32 \\
\hline $\mathrm{UCC}$ & 53,00 & 35,00 & 12,00 & 19,00 & 14,00 & 52,00 & 2,00 & 1,40 & 0,06 & 0,10 & 0,30 & 17,00 & 10,00 & 2,50 \\
\hline A & 7,08 & 7,31 & 3,01 & 3,47 & 1,51 & 11,18 & 0,75 & 0,14 & 0,05 & 0,05 & 0,06 & 2,40 & 0,70 & 0,36 \\
\hline
\end{tabular}

UCC (Upper Continental Crust ou concentrations métalliques crustales), A (bruit de fond de la lagune)

Tableau 4 : Moyennes des fractions $\mathrm{HCl}$ et résiduelle par rapport à la teneur totale.

\begin{tabular}{lccccc}
\hline & $\begin{array}{c}\text { Teneurs totales } \\
(\mathbf{m g} / \mathbf{k g})\end{array}$ & \multicolumn{2}{c}{ Teneurs HCl } & \multicolumn{2}{c}{ Teneurs Résiduelles } \\
\cline { 3 - 6 } & $(\mathbf{m g} / \mathbf{k g})$ & $\mathbf{( \% )}$ & $\mathbf{( m g / k g )}$ & $\mathbf{( \% )}$ \\
\hline $\mathrm{V}$ & 48,20 & 20,20 & 41,99 & 28,00 & 58,01 \\
$\mathrm{Cr}$ & 223,10 & 63,90 & 28,64 & 159,20 & 71,36 \\
$\mathrm{Co}$ & 7,50 & 3,50 & 46,50 & 4,00 & 53,50 \\
$\mathrm{Ni}$ & 20,00 & 9,50 & 47,45 & 10,50 & 52,55 \\
$\mathrm{Cu}$ & 25,70 & 10,60 & 41,28 & 15,50 & 58,72 \\
$\mathrm{Zn}$ & 146,30 & 65,60 & 44,82 & 80,70 & 55,18 \\
$\mathrm{As}$ & 5,70 & 2,00 & 34,90 & 3,70 & 65,10 \\
$\mathrm{Mo}$ & 8,30 & 2,40 & 28,68 & 5,90 & 71,32 \\
$\mathrm{Ag}$ & 0,50 & 0,20 & 34,46 & 0,30 & 65,54 \\
$\mathrm{Cd}$ & 0,50 & 0,30 & 54,71 & 0,20 & 45,29 \\
$\mathrm{Sb}$ & 1,60 & 0,03 & 1,87 & 1,50 & 98,13 \\
$\mathrm{~Pb}$ & 48,90 & 11,10 & 22,76 & 37,70 & 77,24 \\
$\mathrm{Th}$ & 3,30 & 1,10 & 32,79 & 2,20 & 67,21 \\
$\mathrm{U}$ & 3,30 & 1,80 & 53,68 & 1,50 & 46,32 \\
\hline
\end{tabular}


Tableau 5 : Moyenne $(\%)$ de chacune des fractions par rapport à la teneur totale.

\begin{tabular}{|c|c|c|c|c|}
\hline & F1 & F2 & F3 & $\mathbf{R}$ \\
\hline V & 1,52 & 3,45 & 15,23 & 79,80 \\
\hline $\mathrm{Cr}$ & 3,73 & 18,14 & 4,27 & 73,86 \\
\hline Co & 9,66 & 7,24 & 13,07 & 70,04 \\
\hline $\mathrm{Ni}$ & 13,41 & 12,11 & 0,00 & 74,48 \\
\hline $\mathrm{Cu}$ & 2,30 & 3,12 & 12,96 & 81,62 \\
\hline $\mathrm{Zn}$ & 1,52 & 3,45 & 15,23 & 79,80 \\
\hline As & 5,14 & 15,26 & 3,11 & 76,49 \\
\hline Mo & 6,92 & 38,41 & 6,90 & 47,78 \\
\hline $\mathrm{Sb}$ & 3,75 & 6,99 & 0,90 & 88,67 \\
\hline $\mathrm{Pb}$ & 11,81 & & 1,01 & 77,24 \\
\hline Th & 6,85 & 28,98 & 4,14 & 60,03 \\
\hline
\end{tabular}

\section{DISCUSSION}

Teneurs totales en ETM

Les teneurs en métaux des sédiments de la baie de Biétry sont présentées dans le Tableau 3, conjointement avec les concentrations métalliques crustales (Wedephol, 1995). Les sédiments de cette baie présentent de fortes concentrations. D'une manière générale, les teneurs moyennes sont supérieures aux valeurs d'UCC (Upper Continental Crust). Comparativement aux résultats de l'échantillon $\mathrm{A}$ (bruit de fond), les concentrations minimales dans la baie de Biétry sont supérieures pour un même élément considéré. L'analyse de ce tableau, fait ressortir l'existence d'une pollution, tant d'un point de vue général (valeurs d'UCC), que d'un point de vue local (échantillon A).

\section{Évaluation du niveau de pollution}

L'évaluation du niveau de pollution de la baie de Biétry (Figure 2) fait apparaître les 6 classes de l'Igeo de Müller (1981). Ces métaux peuvent être classés en deux (2) groupes :

(i) non pollués (classe 0 et 1 ) : Ce sont: Th, $\mathrm{Co}, \mathrm{V}, \mathrm{Ni}, \mathrm{Ag}$ et $\mathrm{Sb}$, dont les proportions varient de 55 à $70 \%$. (ii) pollués (classes 2, 3, 5, 6) : Ils sont dans une proportion variant de 100 à $70 \%$. Il s'agit de $\mathrm{Cr}$, Mo, $\mathrm{As}, \mathrm{U}, \mathrm{Pb}, \mathrm{Cd}, \mathrm{Zn}$ et $\mathrm{Cu}$.

Cette classification empirique (en éléments non pollués et pollués) permet une approche plus quantitative et qualitative de l'estimation du niveau de pollution dans la baie de Biétry. Toutefois, les données de concentrations totales des métaux, bien que nécessaires, ne nous informent pas sur la disponibilité et la mobilité de chaque élément trace. Or selon plusieurs études (Förstner et al., 1986), connaître les seules concentrations totales des polluants est insuffisant pour évaluer les impacts environnementaux des sédiments contaminés. En effet, la mobilité potentielle, les comportements chimiques des éléments traces et leur toxicité biologique dépendent avant tout de leur spéciation (forme chimique et/ou localisation) dans les sédiments.

\section{Biodisponibilité des ETM}

L'extraction au $\mathrm{HCl}(1 \mathrm{~N})$ est indiquée empiriquement dans certaines études pour extraire une partie des métaux potentiellement biodisponibles et donc qui présentent un risque éventuel pour le biota (Langston et al., 1999). En outre, dans les systèmes aquatiques 
peu ou pas pollués, les ETM sont typiquement trouvés dans la fraction résiduelle (la matrice cristalline) des sédiments de surface, alors que dans les systèmes soumis à des pollutions anthropiques, les mêmes métaux seront associés à la fraction réactive des particules (Gibbs, 1973). Les ETM liés à cette fraction réactive peuvent être solubilisés par attaque $\mathrm{HCl}$ 1N (Morse et Luther III, 1999).

La Figure 3 présente la contribution moyenne des métaux de la fraction $\mathrm{HCl}$ (exprimée en \%) à la teneur totale. Les concentrations d'ETM de la fraction extraite par $\mathrm{HCl}$ sont reportées dans le Tableau 4. $\mathrm{Ni}_{\mathrm{HCl}}, \mathrm{Co}_{\mathrm{HCl}}$ et $\mathrm{Zn}_{\mathrm{HCl}}$ représentent un peu plus de $45 \%$ de la fraction totale. Les autres métaux présentent une fraction $\mathrm{HCl}$ moins importante dans l'ordre décroissant suivant : $\mathrm{V}>\mathrm{Cu}>\mathrm{As}>\mathrm{Th}>\mathrm{Mo}>\mathrm{Pb}$ et $\mathrm{Sb}$. Ainsi, les fortes proportions d'ETM associées à la phase réactive suggèrent une biodisponibilité potentielle pour $\mathrm{Ni}$, Co et $\mathrm{Zn}$, et dans une moindre mesure $\mathrm{V}, \mathrm{Cu}, \mathrm{As}, \mathrm{Th}, \mathrm{Mo}, \mathrm{Pb}$ et $\mathrm{Sb}$.

\section{Phases porteuses et potentiel de mobilité des ETM}

La répartition géochimique des métaux dans les différentes fractions opérationnellement définies est donnée dans le Tableau 5. Pour chaque métal, est reportée la contribution moyenne (\%) de chacune des fractions par rapport à la teneur totale (Figure 4). Ce mode de représentation permet d'une part, une vision globale de la spéciation particulaire des éléments traces métalliques ; d'autre part, une mise en évidence des différences de réactivité entre les métaux. La fraction résiduelle est obtenue en sommant les proportions des fractions acétate (F1), ascorbate (F2) et $\mathrm{H}_{2} \mathrm{O}_{2}(\mathrm{~F} 3)$.

Dans la baie de Biétry, la spéciation des ETM est dominée par la fraction résiduelle (au moins supérieure à 60\%), exception faite du $\mathrm{Zn}$ et de Mo qui montrent des fractions résiduelles relativement faibles (respectivement de 32 et $48 \%$ de la teneur totale). Néanmoins, compte tenu des proportions de chaque phase non résiduelle, nous pouvons affirmer que c'est plutôt Zn qui présente le plus grand potentiel de mobilité et de disponibilité. En effet, dans les $68 \%$ de part non résiduelle, le zinc apparaît principalement sous forme carbonate/échangeable (33\%). C'est la fraction échangeable la plus significative, tandis que le molybdène est associé aux oxyhydroxydes de Fe et Mn avec 39\%. Or selon Leleyter et Probst (1999), parmi les éléments associés aux phases non résiduelles, ceux liés aux fractions échangeables et carbonatées sont plus facilement extractibles face aux changements de conditions physico-chimiques de l'environnement et donc plus mobiles et potentiellement plus toxiques que ceux liés aux phases réductibles et oxydables.

Ainsi, en tenant compte de la proportion totale des phases non résiduelles, mais également de la part de chaque fraction non résiduelle, nous affirmons que la disponibilité et la mobilité des ETM dans les sédiments de la baie de Biétry évolue dans l'ordre suivant, en allant du plus mobile vers le moins mobile: $\mathrm{Zn}>\mathrm{Ni}>\mathrm{Pb}>\mathrm{Co}>\mathrm{Th}>\mathrm{Mo}>$ As $>\mathrm{Sb}>\mathrm{V}>\mathrm{Cr}$ (Figure 5).

\section{Conclusion}

Les concentrations métalliques de la croûte continentale sont inférieures à celles des sédiments de la baie de Biétry. Ces anomalies métalliques s'amplifient lorsque, du point de vue régional, elles sont comparées au bruit de fond de la lagune Ebrié.

Cette pollution métallique s'évalue par l'utilisation de l'Igeo de Müller (1981). Les 2/3 des sédiments de cette baie sont modérément à très sévèrement pollués avec un niveau qui varie de 2 à 6 dans la classification de Müller (1981). Les métaux les plus polluants sont, respectivement, par teneurs décroissantes : $\mathrm{Cr}, \mathrm{Mo}, \mathrm{As}, \mathrm{U}, \mathrm{Pb}, \mathrm{Cd}, \mathrm{Zn}$ et $\mathrm{Cu}$.

Le gradient de biodisponibilité dans la baie de Biétry s'établit comme suit: $\mathrm{Ni}>\mathrm{Co}>\mathrm{Zn}$ et dans une moindre mesure $\mathrm{V}>\mathrm{Cu}>\mathrm{As}>\mathrm{Th}>\mathrm{Mo}>\mathrm{Pb}>\mathrm{Sb}$. Dans les phases porteuses des ETM, la fraction résiduelle est 
la plus dominante pour tous les métaux à l'exception de Mo (48\%) et $\mathrm{Zn} \mathrm{(32 \% ).}$ Toutefois, dans la fraction non résiduelle, certaines phases sont relativement plus stables que d'autres. C'est la raison pour laquelle, outre la fraction non résiduelle totale, il faut prendre en compte la part de chaque fraction non résiduelle pour évaluer la mobilité potentielle des polluants dans un environnement. Le degré de mobilité décroissant des ETM polluants s'établit comme suit: $\mathrm{Zn}, \mathrm{Ni}, \mathrm{Pb}, \mathrm{Co}$, Th, Mo, As, Sb, Cr.

\section{REFERENCES}

Alborés AF, Cid BP, Gómez EF, López EF. 2000. Comparison between sequential extraction procedures and single extractions for metal partioning in sewage sludge samples. Analyst, 125: 1353-1357.

Baize D. 1997. Teneurs en éléments traces métalliques dans les sols (France). Références et stratégies d'interprétation. INRA Paris; p. 408.

Batley GE. 1989. Collection, preparation and storage of samples for speciation analysis. In Trace Element Speciation, Analytical Methods and Problems, Batley GE (Ed.). CRC Press Inc: Florida; 1-24.

Farrah H, Pickering WF. 1993. Factors influencing the potential mobility and bioavailability of metals in dried lake sediments. Chem. Spec. Bioavail., 5: 8196.

Filella M, Town R, Buffle J. 1995. Speciation in fresh waters. In Chemical Speciation in the Environment, Ure AM, Davidson CM (eds). Blackie Academic and Professional; 169-200.

Förstner U, Aslf W, Calmano W, Kersten M, Salomons W. 1986. Mobility of heavy metals in degred harbour sediments. In Sediments and Water Interactions, SlyPeter G (ed.). Springer-Verlag: New York, United States; 371-380.

Gibbs RJ. 1973. Mechanism of metal transport in rivers. Science, 180: 274-280.
Gomez-Ariza JL, Morales E, Giraldez I, Sanchez-Rodas D. 2001. Sample treatment and Storage in Speciation Analysis. In Trace Element Speciation for Environment, Food and Health, Ebdon L, Pitts L, Cornelis R, Crews H, Donard OFX, Quevauviller $\mathrm{Ph}$ (eds). Royal Society of Chemistry: Cambridge; 51-80.

Langston WJ, Burt GR, Pope ND. 1999. Bioavailability of metals in sediments of the Dogger Bank (central North Sea): a mesocosm study Estuar. Coast. Shelf Sci., 48 : 519-540.

Leleyter L, Probst JL. 1999. A new sequential extraction procedure for the speciation of particulate trace elements in river sediments. International Journal of Environment Analytical Chemistry, 73: 109-128.

Morse JW, Luther III GW. 1999. Chemical influences on trace metal-sulfide interactions in anoxic sediments. Geochimica \& Cosmochimica Acta, 63: 3373-3379.

Müller G. 1981. Die Schwermetallbelastung der sedimente des Neckars und seiner Nebenflusse: eine Bestandsaufnahme. Chemical Zeitung, 105: 157-164.

Orsini L, Bermond A. 1993. Application of a sequential extraction procedure to calcareous soil samples: Preliminary studies. Int. J. Environ. Anal. Chem., 51: 85-95.

Quevauviller P. 2002. Operationally-defined extraction procedures for soil and sediment analysis. Part 3 : New CRMs for trace-element extractable contents. Trends Anal. Chem., 21: 774-784.

Quevauviller P. 1998. Operationally-defined extraction procedures for soil and sediment analysis. Part 1. Standardization. Trends Anal. Chem., 17: 289-298.

Rosenberg E., Ariese F. 2001. Quality control in speciation analysis. In Trace element Speciation for Environment, Food and Health, Ebdon L, Pitts L, Cornelis R, Crews H, Donard OFX, Quevauviller Ph 
(eds). The Royal Society of Chemistry: Cambridge, UK.

Salvarredy-Aranguren MM, Probst A, Roulet M, Isaure MP. 2008. Contamination of surface waters by mining wastes in the Milluni Valley (Cordillera Real, Bolivia): mineralogical and hydrological influences. Applied Geochemistry, 23 : 1299-1324.

Sigg L, Behra P, Stumm W. 2006. Chimie des Milieux Aquatiques. Chimie des Eaux Naturelles et des Interfaces dans l'Environnement (4 ${ }^{\text {ème }}$ édition). Dunod : Paris ; $564 \mathrm{p}$.

Tack FMG, Vossius HAH, Verloo MG. 1996. A comparison between sediment metal fractions, obtained from sequential extraction and estimated from single samples. Intern. J. Environ. Anal. Chem., 63: 61-66.

Templeton DM, Ariese F, Cornelis R, Danielsson L-G, Muntau H, VAN Leeuwen HP. 2000. IUPAC guidelines for terms related to chemical speciation and fractionation of trace elements. Pure Appl. Chem., 72: 72-143.

Tessier A, Campbell PGC, Bisson M. 1979. Sequential extraction procedure for the speciation of particulate trace metals. Anal. Chem., 51: 844-851.

Wedepohl KH. 1995. The composition of continental crust. Goechimica and Cosmochimica Acta., 59(7): 217-1232. 